\title{
NUEVOS INDICADORES DE PRODUCCIÓN Y TRANSFERENCIA DE CONOCIMIENTO: UNA PERSPECTIVA EN SALUD PÚBLICA
}

\author{
Norma Patricia Navor Galeana ${ }^{1}$ \\ María Guadalupe Calderón Martínez² \\ María Saiz Santos ${ }^{3}$
}

\begin{abstract}
In public health, the production of knowledge and its transfer to clinical practice are two fundamental phases for an innovation process aimed at improving the quality of patient care. It is necessary for health institutions to carry out careful monitoring, measuring these two phases through a set of indicators that are proposed based on an in-depth analysis of innovation and knowledge transfer literature. The contribution of this study is the proposal of translational research indicators in health.
\end{abstract}

Keywords: translational research; innovation indicators; applied research; literature review.

Resumen: En el ámbito de la salud pública, la producción de conocimiento y su transferencia hacia la práctica clínica, son dos fases fundamentales para un proceso de innovación orientado hacia la mejora en la calidad de la atención a los pacientes. Es necesario que las instituciones de salud puedan realizar un seguimiento cuidadoso, midiendo estas dos fases a través de un conjunto de indicadores que se proponen a partir de un análisis en profundidad de la literatura en innovación y transferencia de conocimiento. La contribución de este estudio es la propuesta de indicadores en el ámbito de la salud, en investigación traslacional.

Palabras-claves: investigación traslacional; indicadores de innovación; investigación aplicada; revisión de la literatura.

\footnotetext{
${ }^{1}$ Posgrado en Ciencias de la Administración - UNAM y Departamento de Evaluación Tecnológica del Instituto Nacional de Rehabilitación - LGII - México. Correo electrónico: navorgp@gmail.com

${ }^{2}$ FES Cuautitlán - UNAM - México. Correo electrónico: gcalderon@cuautitlan.unam.mx

${ }^{3}$ Universidad del Pais Vasco - UPV - España. Correo electrónico: maria.saiz@ehu.eus
}

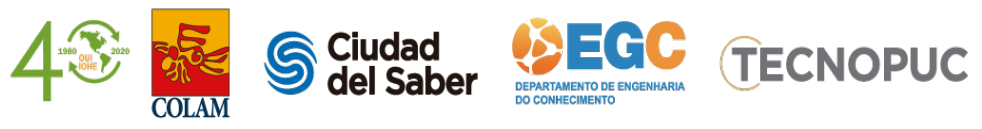




\section{INTRODUCCIÓN}

El Sistema Nacional de Salud en México está integrado por la seguridad social, servicios de salud pública y servicios de salud privada. Los sistemas de seguridad social como el Instituto Mexicano del Seguro Social (IMSS) y el Instituto de Seguridad y Servicios Sociales de los Trabajadores del Estado (ISSSTE) ofrecen diferentes servicios, incluyendo atención médica y pensiones para los trabajadores asalariados en el sector formal.

La Secretaría de la Defensa Nacional (SEDENA), la Secretaría de Marina (SEMAR) y Petróleos Mexicanos (PEMEX) proporcionan servicios similares al IMSS o ISSSTE (Dantés, et al., 2011). Los servicios de salud pública como la Secretaría de Salud (SS), programas especiales del IMSS y el actualmente creado Instituto de Salud para el Bienestar (INSABI) ofrecen atención a la salud a todos los mexicanos que no están cubiertos por los otros esquemas. Estas instituciones públicas ofrecen cobertura a nivel nacional en tres niveles. En el primero, las clínicas asisten a pacientes ambulatorios; en el segundo los hospitales generales y en el tercero los Institutos Nacionales de Salud (INS) y los hospitales de especialidades (Dantés, et al., 2011).

Es en el tercer nivel de atención donde principalmente se lleva a cabo la investigación en salud. El Sistema de Innovación del Sector Salud en México (SISSM), está integrado por estas instituciones y otros Centros Públicos de Investigación (CPI) así como por universidades y empresas. En México se han emitido iniciativas para fomentar la innovación en salud, una de ellas se emitió en 2017 por la Comisión Coordinadora de los Institutos Nacionales de Salud y Hospitales de Alta Especialidad (CCINSHAE) en colaboración con la Universidad Nacional Autónoma de México (UNAM) y el Consejo Nacional de Ciencia y Tecnología (Conacyt) para generar un Consorcio Nacional de Investigación en Medicina Traslacional e Innovación y abrir un espacio para la transición de la investigación básica a aplicaciones clínicas y desarrollos tecnológicos (Gaceta UNAM, 2018).

En 2018 el Conacyt emitió otra iniciativa para la creación de Nodos Binacionales de Innovación (NoBi), reúne a varios grupos de instituciones científico-académicas, cuya labor es

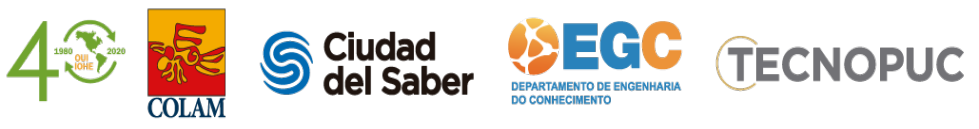


el desarrollo de herramientas y recursos que expandan los beneficios de la innovación y el emprendimiento de base tecnológica, con énfasis en la formación de capacidades del personal científico en temas de innovación. Participan el Instituto Tecnológico de Estudios Superiores de Monterrey (ITESM), el IMSS, la Universidad Autónoma del Estado de Morelos, el Conacyt, The National Science Foundation of U. S. y el Instituto Nacional de Rehabilitación Luis Guillermo Ibarra (INR-LGII). El nodo emplea la metodología I-Corps, esta metodología pretende apoyar a los investigadores que buscan comercializar su tecnología a través de la validación en el mercado y fomentar el emprendimiento de base tecnológica (Conacyt, 2018).

En esta investigación, presenta un estudio para el desarrollo de un marco conceptual. A partir de la revisión sistemática de la literatura en innovación, producción y transferencia de conocimiento, proceso descrito por Massaro et al., (2016), se plantean indicadores de transferencia en el ámbito de la salud pública. La contribución central es la identificación de la importancia de la investigación traslacional en salud.

\section{METODOLOGÍA}

Para identificar los documentos relevantes, se siguió el método utilizado en estudios previos (Dumay y Cai, 2014; Massaro et al., 2015). Los pasos para realizar la revisión sistemática de la literatura de manera replicable (Christoffersen, 2013; Thorpe et al., 2005) fueron cinco: (1) definir las preguntas de investigación, (2) diseñar un protocolo de revisión, (3) determinar los artículos a incluir y llevar a cabo una lectura integral, (4) desarrollar un sistema de términos clave; y (5) analizar de manera crítica y discutir los resultados. Siguiendo a Massaro et al., (2016) y Secundo, Ndou y Del Vecchio (2019), se determinaron las siguientes preguntas para la revisión de la literatura:

1. ¿Cuál es el enfoque de la literatura sobre indicadores en innovación?

2. ¿Cuáles son las implicaciones para la investigación en producción, difusión, diseminación y transferencia de conocimiento en salud?

Se estableció el protocolo de investigación para determinar la fuente de información, los métodos a utilizar, así como los medios y herramientas para analizar y sintetizar los textos

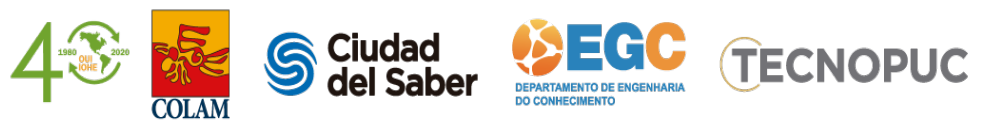


(Petticrew y Roberts, 2008). Para ello se categorizaron los temas en salud pública para tener una comprensión más específica del fenómeno.

\section{RESULTADOS}

La revisión sistemática de la literatura permitió construir un panorama sobre indicadores de innovación y los temas de producción, difusión, diseminación y transferencia de conocimiento, innovación en salud y aspectos metodológicos de la investigación traslacional (IT) para contextualizar los conceptos en el ámbito de la salud. Los siguientes apartados permiten responder a la pregunta uno.

\subsection{INVENCIÓN, INNOVACIÓN Y PROCESO DE INNOVACIÓN}

La invención es la creación de algo nuevo y la innovación es llevar la invención a la práctica. Una innovación es la introducción de un nuevo, o significativamente mejorado producto (bien o servicio), o un proceso, o un nuevo método de comercialización o un nuevo método organizativo, aplicado a las prácticas del negocio, a la organización del trabajo o a las relaciones externas (OCDE, 2019).

El proceso de innovación involucra la exploración y la explotación de productos, procesos o servicios tanto nuevos como mejorados, basados en una ventaja de práctica técnica, o en un cambio en la demanda de mercado, o por una combinación de ambos. Este proceso se compone de tres fases: producción de conocimiento científico y tecnológico, transferencia de conocimiento y respuesta e influencia del mercado (Pavitt, 2005).

El manual de Oslo (2019), aporta directrices para la obtención de datos sobre un proceso de innovación (por ejemplo, las actividades innovadoras, los gastos y los vínculos), aborda también aspectos de cambios significativos en la empresa e incluye factores que influyen en las actividades y los resultados de innovación (Tabla 1). 
Tabla 1 - Indicadores en los procesos de innovación I

\section{Indicadores}

Actividades y gastos en innovación

Factores que influyen en la innovación proceso de innovación

Medición de las actividades científicas y tecnológica

Áreas que deben medirse en el proceso de innovación

\section{Descripción}

a) Actividades científicas, tecnológicas, organizativas, financieras y comerciales. b) se recomienda considerar actividades no incluidas en $\mathrm{I}+\mathrm{D}, \mathrm{c}$ ) actividades de innovación exitosas, en curso y abandonadas, la estimación de los gastos se efectúa considerando la suma de actividades durante un periodo de tiempo dado.

a) Identificar los motivos de la empresa para innovar, b) factores económicos, c) falta de personal experto, d) factores legales e) capacidad de la empresa para apropiarse de las mejoras de sus actividades.

a) Difusión entrante, b) identificar el tipo de vínculo, c) obtener datos de fuentes de información de libre acceso, adquisición de conocimiento y tecnología y cooperación en materia de innovación, d) quién desarrolla la innovación, e) tipos de conocimiento y métodos de transferencia, f) capital social, g) información suplementaria sobre la cooperación en innovación, h) difusión saliente, i) gestión del conocimiento.

a) Dos indicadores principales: recursos dedicados a I+D y patentes, b) un indicador secundario: la bibliometría (estadística sobre publicaciones científicas), incluir los recursos humanos cualificados, balanza de pagos tecnológicos, indicadores de globalización y la actividad en sectores de alta tecnología, c) recogida de datos sobre I+D se basan en el Manual de Frascati (OCDE, 2002), d) incluir progreso técnico, esfuerzos realizados y aprendizaje por la práctica, e) otros manuales y guías para la medición de las actividades científicas y tecnológicas: The Measurement of Human Resources Devoted to S\&T Canberra Manual, Measuring productivity Manual, A Framework for Biotechnology Statistic, f) análisis de la gestión del conocimiento.

a) Los insumos de la innovación, b) los vínculos y el papel de la difusión, c) el impacto de la innovación, d) Incentivos y obstáculos a la innovación, e) La demanda de la innovación, f) recursos humanos, g) legislación y normativa, h) tipo de innovación, i) novedad y difusión.

a) Los componentes y la cobertura de las actividades de innovación, b) investigación y desarrollo experimental, c) actividades relativas a las innovaciones de producto y proceso, d) desarrollo y uso de programas y aplicaciones informáticos en actividades

Actividades de innovación de innovación, e) incluir medición de datos cualitativos de la innovación, f) desglose según el tipo de gasto: costos de mano de obra más otros gastos corrientes, inversiones en capital fijo consagradas a la innovación; g) desglose según el origen de fondos: fondos propios, procedentes de empresas participantes, otras empresas, sociedades financieras, fondos públicos y procedentes de organismos supranacionales e internacionales y otros orígenes.

a) Objetivos y efectos de las innovaciones, b) otras mediciones de los impactos sobre

Objetivos, barreras y resultados de la innovación los resultados de las empresas, c) impacto sobre la cifras de negocios, d) ciclo de vida de los productos, e) innovaciones de proceso, f) innovaciones de mercadotecnia, g)impacto de las innovaciones de proceso en los costos y el empleo, h) impacto de la innovación en la productividad, i) factores que obstaculizan las actividades de innovación, j) cuestiones sobre la apropiación de las innovaciones.

Fuente: Elaboración propia a partir de OCDE (2019)

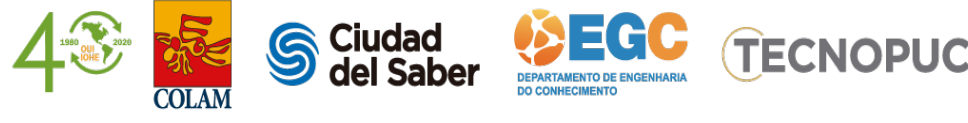


Keith Smith (2005) enfoca la medición de la innovación en la conceptualización, recolección y análisis de las mediciones. La Tabla 2 muestra un resumen de aspectos relevantes del autor.

Tabla 2 - Medición de indicadores en innovación II

\begin{tabular}{|c|c|}
\hline Indicadores & Descripción \\
\hline $\begin{array}{l}\text { El proceso de } \\
\text { innovación no es } \\
\text { secuencial }\end{array}$ & $\begin{array}{l}\text { El proceso involucra iteraciones y retroalimentación en la creación de conocimiento } \\
\text { El proceso de innovación involucra múltiples entradas } \\
\text { La innovación no depende de una invención } \\
\text { El proceso de innovación es multidimensional }\end{array}$ \\
\hline $\begin{array}{l}\text { Indicadores } \\
\text { tecnométricos }\end{array}$ & $\begin{array}{l}\text { Exploran las características del desarrollo tecnológico de los productos (Saviotti } \\
\text { 1996, Saviotti 2001; Grupp 1994, 1998) }\end{array}$ \\
\hline Indicadores sintéticos & Desarrollados por consultores (World Economic Forum, 2003) \\
\hline $\begin{array}{l}\text { Base de datos en } \\
\text { tópicos específicos }\end{array}$ & $\begin{array}{l}\text { Como herramienta de investigación individual o grupal (Patel y Pavitt } 1997 \text { y 1999; } \\
\text { Hagedoorn y Shakenraad 1990; OECD, 2001) }\end{array}$ \\
\hline Datos Bibliométricos & $\begin{array}{l}\text { Análisis de la composición y la dinámica de las publicaciones científicas y las citas, } \\
\text { Science Citation Index y del Institute for Scientific Information database. }\end{array}$ \\
\hline $\begin{array}{l}\text { Estadística e } \\
\text { Indicadores } \mathrm{I}+\mathrm{D} \text {. }\end{array}$ & $\begin{array}{l}\text { Standard Practice for Surveys of Research and Experimental Development, Frascati } \\
\text { Manual. Generación de nuevo conocimiento y nuevas prácticas, considerando tres } \\
\text { categorías: Investigación básica, investigación aplicada y desarrollo experimental. }\end{array}$ \\
\hline $\begin{array}{l}\text { Criterios para los } \\
\text { indicadores de } \mathrm{I}+\mathrm{D}\end{array}$ & $\begin{array}{l}\text { Si } I+D / \text { Producción es }>5 \% \text { es una industria de alta tecnología. } \\
\text { Si } I+D / \text { Producción es }>3 \% \text { y }<5 \% \text { es una industria media de alta tecnología. } \\
\text { Si } I+D / \text { Producción es }>1 \% \text { y }<3 \% \text { es una industria de baja tecnología. } \\
\text { Si } I+D / \text { Producción es }>1 \% \text { y }<0 \% \text { es una industria de muv baia tecnología. }\end{array}$ \\
\hline Datos sobre patentes & $\begin{array}{l}\text { Si } I+D / \text { Produccion es }>1 \% \text { y }<0 \% \text { es una industria de muy baja tecnologia. } \\
\text { Se conceden patentes para la tecnología inventiva con promesa comercial. } \\
\text { US Patent office, European Patent Office y el Sistema de Información de la Gaceta } \\
\text { de la Propiedad Industrial (SIGA) del IMPI. }\end{array}$ \\
\hline $\begin{array}{l}\text { Indicadores de } \\
\text { innovación }\end{array}$ & $\begin{array}{l}\text { Base de datos de la Science Policy Research Unit (SPRU) de la Universidad de } \\
\text { Sussex. Información de la innovación tecnológica en la industria británica. }\end{array}$ \\
\hline
\end{tabular}

Fuente: Elaboración propia a partir de (Smith, 2005)

Dentro de las contibuciones teóricas sobre indicadores de innovación, podemos identificar diversos enfoques, así como elementos que definen los procesos para la innovación, destacando aspectos como financiamiento, infraestructura, personas, elementos organizacionales e institucionales, metodologías, alcances y sobre todo la definición de objetivos y alcances de la innovación por parte de las organizaciones. Esta lectura ha permitido 
identificar brechas y espacios existentes en la explicación de la transferencia de conocimiento, entre los resultados de la investigación científica básica y su traducción a innovaciones en salud.

\subsection{PRODUCCIÓN, DIFUSIÓN, DISEMINACIÓN Y TRANSFERENCIA DE CONOCIMIENTO EN SALUD}

En esta sección se presentan los hallazgos identificados a partir de la segunda pregunta planteada. Las entidades de atención a la salud son organizaciones complejas debido a la heterogeneidad de sus profesionales, las redes compuestas y los procesos de toma de decisiones (Karamitri et al., 2017). La producción de conocimiento ha sido estudiada desde el ámbito empresarial por Nonaka y Takeuchi (1995), Smith (2001) y Collins (2010). El conocimiento es un activo intangible en las organizaciones y proporciona una ventaja comparativa.

Específicamente Nonaka \& Takeuchi (1995) definieron cuatro modos de conocimiento: tácito a tácito (socialización), tácito a explícito (externalización), explícito a explícito (combinación) y explícito a tácito (internalización). En el ámbito de la ciencia y tecnología así como en las ciencias sociales, Gibbons et al., (1997) han descrito dos modos de producción de conocimiento: el modo I integra agendas definidas por investigadores, investigación básica vs aplicada, transferencia de resultados unidireccionales a posteriori, disciplinar, mérito científico evaluado por pares, revista científica, recursos públicos, planificación central basada en oferta. El modo II integra redes de colaboración, agendas definidas en contexto de aplicación, solución de problemas, interacción multidireccional durante el proceso de investigación, transdisciplinar, mérito y relevancia evaluado por pares, múltiples medios, diversidad de fuentes, creación de espacios de interacción.

En un estudio realizado por Estabrooks et al., (2006) se muestran algunas teorías de transferencia de conocimiento desde diferentes perspectivas como: organizacional, ciencias sociales, enfermería y promoción a la salud. Una aportación que realiza este autor al marco de referencia para la utilización de las investigaciones en el ámbito de la salud es el modelo de Ottawa (Ottawa Model of Research Use, OMRU; Logan y Graham, 1998). Estabrooks et al., (2008), identificaron las actividades de transferencia de conocimiento de investigadores del

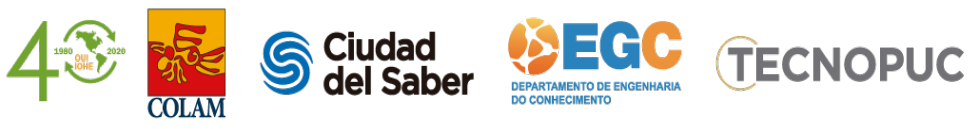


sector salud en diferentes disciplinas y facultades. En este estudio, el modo I se caracterizó por una investigación basada en la curiosidad y en una epistemología positivista (ciencia tradicional) y el modo II se basó en una epistemología de resolución de problemas.

Gagnon (2011), describe las fases de difusión, diseminación e implementación del conocimiento. La difusión de conocimiento se lleva a cabo a través de modelos conceptuales, procesos de intercambio, publicación en revistas científicas, presentación de los resultados en foros e intercambio de conocimiento. Mientras la diseminación se realiza mediante la adaptación de los resultados científicos a un público específico, enlaces de investigadores o usuarios del conocimiento e intercambios y a implementación es un proceso activo de esfuerzos sistemáticos para fomentar la adopción de hallazgos científicos.

La investigación traslacional (IT) ha sido definida como traducción, diseminación y transferencia de conocimiento (Cabieses y Espinoza, 2011), este proceso describe un paradigma que pretende explicar y reducir la distancia existente entre el conocimiento obtenido en el laboratorio y su incidencia en los pacientes (D' Este y Llopis, 2014). Otros autores han definido la IT como la investigación aplicada que pretende traducir el conocimiento científico (Benedyk, et al., 2009; Cabieses y Espinoza, 2011; Kerner, 2006; Venkat et al., 2000).

Cabieses y Espinoza (2011) mencionan tres etapas principales en la IT, T1 “del laboratorio a la cabecera del paciente", T2 "de la investigación a la práctica" y T3 “investigación de nivel político y de impacto sanitario". El proceso de innovación puede ser abordado a partir de las primeras dos fases del proceso de innovación (Pavitt, 2005). En la revisión de textos se detectaron los ejes principales de estudio que se ilustran en la Figura1.

Resaltan las estrategias para la transferencia de conocimiento (Estabrooks et al., 2006; Logan y Graham, 1998; Estabrooks et al., 2008 y Gagnon 2011), así como las etapas de la investigación traslacional (Cabieses y Espinoza, 2011) donde la integración de la IT hasta la etapa T2 permite estudiar la transferencia de los resultados de investigación en salud y por último la identificación de los elementos que limitan la transferencia de los resultados de investigación científica. 
Figura 1 Constructo para la generación de indicadores

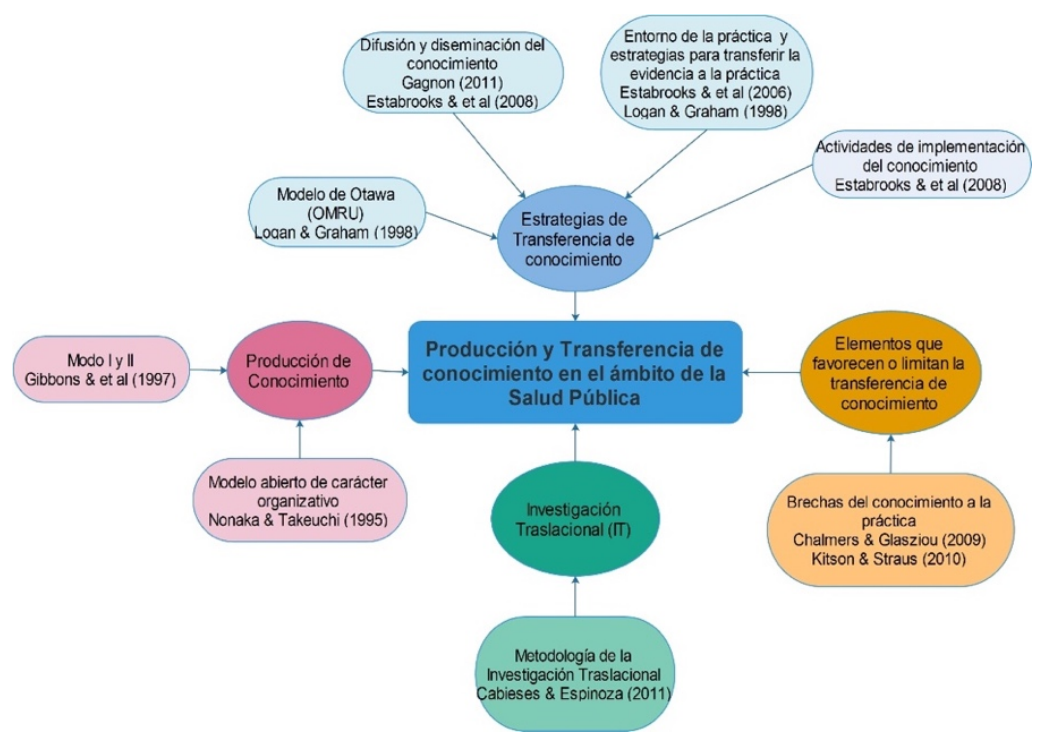

Fuente: Elaboración propia

\section{DISCUSIÓN E IMPLICACIONES SOBRE PRODUCCIÓN, DIFUSIÓN, DISEMINACIÓN Y TRANSFERENCIA DE CONOCIMIENTO EN SALUD}

En esta sección se utilizaron los términos clave identificados por las autoras. De acuerdo con Silverman (2013, p. 275), la delimitación de términos clave permite el análisis y clasificación de la literatura. La transferencia de resultados de investigación científica y tecnológica puede ser estudiada a partir de la integración de:

- Estrategias para la transferencia de conocimiento (Estabrooks et al., 2006; Logan y Graham, 1998; Estabrooks et al., 2008 y Gagnon 2011).

- Investigación Traslacional hasta la etapa T2 (Cabieses y Espinoza, 2011). La integración de la IT hasta la etapa T2 permite estudiar la transferencia de los resultados de investigación en salud desde la investigación básica hasta su aplicación a los pacientes.

- Identificación de los elementos que limitan la transferencia de los resultados de investigación científica y tecnológica hacia la atención médica, análisis de la brecha (Chalmers y Glasziou, 2009; Kitson y Straus, 2010).

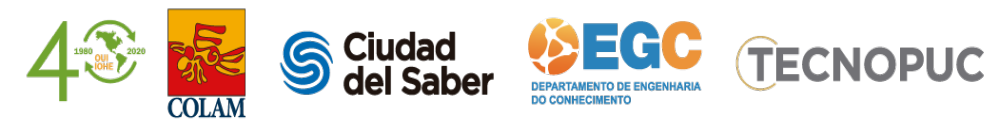


Mediante este análisis y el constructo de la figura 1, se clasificaron los documentos en las tres áreas definidas. Mediante una lectura en profundidad se sintetizó el cuerpo de la literatura y con base en la experiencia de las autoras se identificó la propuesta de indicadores contextualizados al ámbito de la salud pública como se muestran en la Tabla 3.

Tabla 3 - Indicadores en el ámbito de la salud pública

\begin{tabular}{|c|c|c|}
\hline Dimensión & Temas & Indicadores \\
\hline \multirow{5}{*}{$\begin{array}{l}\text { Producción de } \\
\text { conocimiento }\end{array}$} & $\begin{array}{l}\text { Producción de conocimiento Modo I y } \\
\text { Modo II }\end{array}$ & $\begin{array}{l}\text { 1. Area de investigación del personal } \\
\text { 2. Grupo de investigación del personal } \\
\text { 3. Financiamiento a los proyectos de } \\
\text { investigación }\end{array}$ \\
\hline & $\begin{array}{l}\text { En la socialización, los individuos } \\
\text { comparten experiencias, se crea } \\
\text { conocimiento tácito que se comparte } \\
\text { como modelos mentales y habilidades } \\
\text { técnicas }\end{array}$ & $\begin{array}{l}\text { 4. Evaluación de protocolos de } \\
\text { investigación por los comités de ética y de } \\
\text { investigación }\end{array}$ \\
\hline & $\begin{array}{l}\text { La externalización es un proceso que se } \\
\text { realiza con el uso de metáforas, } \\
\text { analogías, conceptos, hipótesis o modelo } \\
\text { y se desarrolla mediante el diálogo y la } \\
\text { reflexión colectiva }\end{array}$ & $\begin{array}{l}\text { 5. Entrenamiento informal para el personal y } \\
\text { estudiantes } \\
\text { 6. Diálogo creativo e intercambio de ideas } \\
\text { en todos los niveles de la institución de salud }\end{array}$ \\
\hline & $\begin{array}{l}\text { La combinación se puede realizar } \\
\text { mediante procedimientos formales }\end{array}$ & $\begin{array}{l}\text { 7. Generación de bases de datos durante el } \\
\text { desarrollo de las investigaciones }\end{array}$ \\
\hline & $\begin{array}{l}\text { La internalización está íntimamente } \\
\text { relacionada con el "aprender haciendo" }\end{array}$ & $\begin{array}{l}\text { 8. Capacitación o entrenamiento formal } \\
\text { 9. Capacitación externa } \\
\text { 10. Simulación y experimentación en áreas } \\
\text { de investigación con métodos y } \\
\text { procedimientos de otros colegas. } \\
\text { 11. Simulación o experimentación en áreas } \\
\text { de investigación con manuales y literatura }\end{array}$ \\
\hline \multirow[b]{3}{*}{$\begin{array}{l}\text { Estrategias de } \\
\text { transferencia de } \\
\text { conocimiento }\end{array}$} & Actividades de difusión y diseminación & $\begin{array}{l}\text { 12. Difusión de sus resultados de } \\
\text { investigación }\end{array}$ \\
\hline & $\begin{array}{l}\text { Modelo de Ottawa (Ottawa Model of } \\
\text { Research Use, OMRU) }\end{array}$ & $\begin{array}{l}\text { 13. Difusión de sus resultados de } \\
\text { investigación en radio, televisión, periódicos } \\
\text { o redes sociales }\end{array}$ \\
\hline & $\begin{array}{l}\text { Actividades de implementación del } \\
\text { conocimiento }\end{array}$ & $\begin{array}{l}\text { 14. Solicitudes de derechos de autor } \\
\text { 15. Solicitudes de patentes } \\
\text { 16. Contribuciones al desarrollo de prácticas } \\
\text { clínicas, mejoras de procesos o manual de } \\
\text { procedimientos } \\
\text { 17. Subcontratación o creación de oficinas } \\
\text { de transferencia }\end{array}$ \\
\hline
\end{tabular}


Temas

Actividades de implementación del conocimiento

Estrategias de transferencia de conocimiento
Razones principales para explicar aquellos fracasos en las etapas tempranas de investigación: la primera razón es que se considera una hipótesis de baja prioridad, la segunda, se evalúa bibliografía de hallazgos científicos poco relevantes y la tercera, los pacientes y médicos no son consultados

Elementos que favorecen $o$ limitan la transferencia de conocimiento
Algunos puntos clave para el estudio de la brecha son: a) identificar las brechas del conocimiento a la práctica, b) los análisis de brechas deben involucrar el uso de métodos rigurosos y comprometer a las partes interesadas más relevantes, c) las estrategias para completar las evaluaciones de necesidades dependen del propósito de la evaluación, el tipo de datos y los recursos disponibles, d) las necesidades pueden ser evaluadas desde la población, una organización o un proveedor de atención médica Investigación aplicada que pretende traducir el conocimiento científico disponible

\section{Indicadores}

18. Colaboración con otras instituciones para la protección de propiedad intelectual 19. Conocimiento del concepto de transferencia de conocimiento, tecnología o resultados de investigación 20. Conocimiento de las políticas institucionales para la transferencia de conocimiento, tecnología o resultados de investigación

21. Motivación del personal por hacer transferencia de conocimiento, tecnología o resultados de investigación

22. Infraestructura institucional para la transferencia de conocimiento, tecnología o resultados de investigación

23. Fomento institucional para la revisión de patentes como antecedente a las propuestas de investigación

24. Fomento institucional para que el personal tenga conocimiento de los problemas de la práctica clínica 25. Fomento institucional para que el personal tenga conocimiento de los problemas de otras instituciones o empresas 26. Iniciativa del personal por realizar búsqueda de patentes como antecedentes a las propuestas de investigación

27. Investigaciones que brindan solución a problemas específicos de la práctica clínica 28. Investigaciones que brindan solución a problemas específicos de otras instituciones o empresas

29. Marco regulatorio institucional alineado para fomentar la transferencia 30. Iniciativa del personal por transferir conocimiento, tecnología o resultados de investigación a la práctica clínica

31. Conocimiento de los conceptos de medicina o investigación traslacional

Investigación
traslacional

Fuente: Elaboración propia

Esta revisión de literatura ha permitido definir la IT en salud y reconocer su complejidad. A partir de esta revisión, encontramos que los factores de análisis en el fenómeno de producción y tansferencia de conocimiento se concentran en: Producción del conocimiento, Investigación Traslacional, y la Brecha del conocimiento a la práctica y las Estrategias de 
transferencia. Estos factores permiten proponer un constructo, el cual puede ser un punto de partida para diseñar instrumentos de carácter cuantitativo para recoger información, o bien para el uso de otras herramientas cualitativas para un estudio en mayor profundidad.

\section{CONCLUSIONES}

La complejidad y especialización de la investigación en salud, ha traido consigo cambios en los intereses y habilidades de los investigadores, generando la necesidad de científicos con un conjunto de habilidades y expertise específico. Este tipo de investigación surge a partir de un área de oportunidad en el planteamiento de mejores modelos para explicar la producción y transferencia de conocimiento en salud, que a diferencia de los modelos lineales suponían una simplicidad en la implementación de los resultados de investigación en salud.

Esta fase de revisión de la literatura, ha permitido generar un marco conceptual e indicadores de análisis para un fenómeno complejo. Sin embargo, una de las limitaciones de esta etapa es la posible subjetividad en la clasificación de los indicadores, en virtud de que el análisis sistemático de la literatura fue realizado mediante la lectura y análisis por parte de las autoras. Con estos resultados, en una siguiente fase será posible utilizar herramientas de análisis de contenido para incorporar otro tipo de indicadores como palabras clave de las publicaciones o citas recibidas.

Este trabajo presenta una propuesta de indicadores contextualizados para la evaluación de la producción y transferencia de conocimiento en el ámbito de la salud pública. Es el resultado de un proceso de análisis de la literatura y los indicadores propuestos enmarcan tanto a las instituciones de salud como al personal que realiza actividades de investigación, desarrollo e innovación, esto hace posible una coevaluación, coproyección y coproducción de iniciativas organizacionales hacia el fortalecimiento de la innovación en salud.

Como futuras líneas de investigación, el análisis permite proponer la incorporación de herramientas de análisis para una doble prueba del modelo propuesto. Así como la construcción de un instrumento para la recolección de datos en instituciones de salud pública. Entre los temas que pueden utilizar este modelo, se identifican: a) difusión de la formación emprendedora en el ámbito de la educación médica, b) intervención de diversos grupos de interés para contribuir a

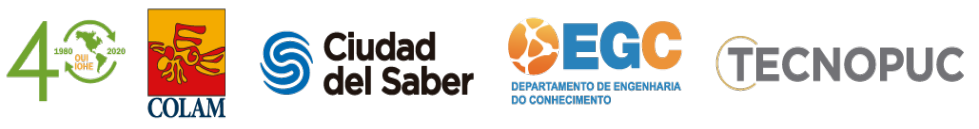


generar procesos de transferencia, c) los flujos del conocimiento desde la investigación hacia otros sectores, d) tecnologías y estrategias para la creación de conocimiento sobre investigación traslacional en salud, e) análisis comparativo de experiencias entre países que han asumido los procesos de transferencia en el sector salud, f) evidencia sobre mejores prácticas y g) análisis desde enfoques multidisciplinarios. Los estudios sobre estos y otros temas podrán derivar en contribuciones teóricas y empíricas, superando las limitaciones que aún caracterizan el debate en investigación traslacional.

\section{AGRADECIMIENTOS}

Agradecemos el apoyo recibido del proyecto UNAM-DGAPA-PAPIIT-IN306320. Administración del conocimiento en investigación traslacional en salud desde el modelo de cadena de valor.

\section{REFERENCIAS}

Benedyk, M., Berglund, L., Facciotti, M., Mobley, W., \& Zucker, D. (2009). Strategies for innovation and interdisciplinary translational research: research and career benefits and barriers. J Investig Med, 57(2), 477-81. http://dx.doi.org/10.2310/JIM.0b013e31819824be

Cabieses, B., \& Espinoza, M. (2011). La investigación traslacional y su aporte para la toma de decisiones en políticas de salud. Rev Peru Med Exp Salud Publica, 28(2), 288-97. https://doi.org/10.17843/rpmesp.2011.282.496

Chalmers, I., \& Glasziou , P. (2009). Avoidable waste in the production and reporting of research evidence. The Lancet, 374(9683), 86-89. https://doi.org/10.1016/S01406736(09)60329-9

Christoffersen, J. (2013). A review of antecedents of international strategic alliance performance: synthesized evidence and new directions for core constructs, 
International Journal of Management Reviews, 15(1), 66-85.

https://doi.org/10.1111/j.1468-2370.2012.00335.x

Collins, H. (2010). Tacit and Explicit Knowledge. Chicago: The University of Chicago Press. Library of Congress Cataloging in Publication Data.

D’Este P. \& Llopis, O. (2014). Investigación traslacional e innovación médica: el caso de las redes CIBER. Revista de la Sociedad Española de Bioquímica y Biología Molecular, 180, 13-16.

Dantés, O. G., Sesma, S., Becerril, V. M., Knaul, F. M., Arreola, H., \& Frenk, J. (2011).

Sistema de salud de México. Salud Pública de México, 53, s220-s232.

https://www.saludpublica.mx/index.php/spm/article/view/5043

Dumay, J. \& Cai, L. (2014). A review and critique of content analysis as a methodology for inquiring into IC disclosure. Journal of Intellectual Capital, 15(2), 264-290.

https://doi.org/10.1108/JIC-01-2014-0010

Estabrooks, C. A., Norton, P., Birdsell, J. M., Newton, M. S., Adewale, A. J., \& Thornley, R. (2008). Knowledge translation and research careers: Mode I and Mode II activity among health researchers. Research Policy, 37(6-7), 1066-1078.

https://doi.org/10.1016/j.respol.2008.04.006

Estabrooks, C., Thompson, D., Lovely, J., \& Hofmeyer, A. (2006). A guide to knowledge translation theory. Journal of Continuing Education in the Health Professions, 26(1), 25-36. https://doi-org.pbidi.unam.mx:2443/10.1002/chp.48

Gaceta UNAM. (2018). Consorcio Nacional de Investigación en Medicina Traslacional e Innovación. Recuperado de https://www.gaceta.unam.mx/tag/consorcio-nacional-deinvestigacion-en-medicina-traslacional-e-innovacion/

Gagnon, M. L. (2011). Moving knowledge to action through dissemination and exchange. Journal of Clinical Epidemiology, 64(1), 25-31. https://doi.org/10.1016/j.jclinepi.2009.08.013

Gibbons, M., Limoges, C., Nowotny, H., Schwartzman, S., Scott, P., \& Trow, M. (1997). La nueva producción de conocimiento. En J. Batallé, (Ed.) La dinámica de la ciencia y la 
investigación en las sociedades contemporáneas. Barcelona: Ediciones PomaresCorredor, S. A.

Karamitri, I., Talias, M., \& Bellali, T. (2017). Knowledge management practices in healthcare settings: a systematic review. Int J Health Plann Mgmt, 32(1), 4-18.

https://doi.org/10.1002/hpm.2303

Kerner, J. F. (2006). Knowledge translation versus knowledge integration: a "funder's" perspective. J Contin Educ Health Prof, 26(1), 72-80. doi: 10.1002/chp.53

Kitson, A., \& Straus, S. E. (2010). The knowledge-to-action cycle: identifying the gaps. CMAJ, E73-E77. https://doi.org/10.1503/cmaj.081231

Logan, J. O., \& Graham, I. D. (1998). Toward a comprehensive interdisciplinary model of health care research use. Sci Commun, 20(2), 227-246. https://doi.org/10.1177/1075547098020002004

Massaro, M., Dumay, J. \& Garlatti, A. (2015). Public sector knowledge management: a structured literature review, Journal of Knowledge Management, 19(3), 530-558. https://doi.org/10.1108/JKM-11-2014-0466

Massaro, M., Dumay, J. \& Guthrie, J. (2016), On the shoulders of giants: undertaking a structured literature review in accounting. Accounting, Auditing \& Accountability Journal, 29(5), 767-801. https://doi.org/10.1108/AAAJ-01-2015-1939

Nodos Binacionales de Innovación (NoBi) - Conacyt. (2018). Recuperado de https://www.conacyt.gob.mx/index.php/el-conacyt/organigrama/direccion-adjunta-dedesarrollo-tecnologico-e-innovacion/nodos-binacionales-de-innovacion-nobi

Nonaka, I., \& Takeuchi, H. (1995). The Knowledge-Creating Company: How Japanese Companies Create the Dynamics of Innovation. New York, NY: Oxford University Press.

OCDE. (2002). Manual de Frascati. Propuesta de Norma Práctica para Encuestas de Investigación y Desarrollo Experimental. Fundación Española para la Ciencia y la Tecnología (FECYT). Recuperado de https://www.fecyt.es/es/publicacion/manual-defrascati-2002 
OCDE. (2019). Oslo Manual 2018. Guidelines for Collecting, Reporting and Using Data on Innovation. París: OCDE Publishing. Recuperado de http://www.oecd.org/science/oslo-manual-2018-9789264304604-en.htm

Pavitt, K. (2005). Innovation Process. En J. Fagerberg, D. C. Mowery, \& R. R. Nelson (Eds.), The Oxford handbook of innovation. New York: Oxford university press. pp. 86-114.

Petticrew, M. \& Roberts, H. (2008). Systematic Reviews in the Social Sciences: A Practical Guide. New York: John Wiley \& Sons.

Secundo, G., Ndou, V., Del Vecchio, P. and De Pascale, G. (2019), Knowledge management in entrepreneurial universities: A structured literature review and avenue for future research agenda, Management Decision, 57(12), 3226-3257. https://doi.org/10.1108/MD-11-2018-1266

Silverman, D. (2013). Doing Qualitative Research. London: Sage Publications

Smith, E. (2001). The role of tacit and explicit knowledge in workplace. J Knowl Manag, 5(4), 311-321. https://doi.org/10.1108/13673270110411733

Smith, K. (2005). Measuring Innovation. En J. Fagerberg, D. C. Mowery, \& R. R. Nelson (Eds.), The Oxford handbook of innovation. New York: Oxford University Press. pp. 148-177.

Thorpe, R., Holt, R., Macpherson, A. \& Pittaway, L. (2005). Using knowledge within small and medium-sized firms: a systematic review of the evidence, International Journal of Management Reviews, 7(4), 257-281. https://doi.org/10.1111/j.1468$\underline{2370.2005 .00116 . x}$

Venkat N., K. M., Gregg, E. W., Engelgau, M. M., Moore, B., Thompson, T. J., Williamson, D. F., \& Vinicor, F. (2000). Translation research for chronic disease: the case of diabetes. Diabetes Care, 23(12), 1794-1798.

https://doi.org/10.2337/diacare.23.12.1794 